\title{
Exchange interaction in the heavy rare earth metals calculated from energy bands
}

\author{
Lindgård, Per-Anker
}

Publication date:

1974

Document Version

Publisher's PDF, also known as Version of record

Link back to DTU Orbit

Citation (APA):

Lindgård, P-A. (1974). Exchange interaction in the heavy rare earth metals calculated from energy bands. Risø National Laboratory. Risø-M No. 1701

\section{General rights}

Copyright and moral rights for the publications made accessible in the public portal are retained by the authors and/or other copyright owners and it is a condition of accessing publications that users recognise and abide by the legal requirements associated with these rights.

- Users may download and print one copy of any publication from the public portal for the purpose of private study or research.

- You may not further distribute the material or use it for any profit-making activity or commercial gain

- You may freely distribute the URL identifying the publication in the public portal

If you believe that this document breaches copyright please contact us providing details, and we will remove access to the work immediately and investigate your claim 
Title and author(s)

Exchange Interaction in the Heavy Rare Earth Metals Calculated from Energy Bands by Per-Anker Lindgärd
Date Fesciany :27.

Department or group

Physios

Group's own registration number(s)

The indirect exchange interaction (RKKY) is calculated for the heavy rare earth metals. The conduction electrons are treated by the argumented-plane-wave (APW) method. Numerical results for the susceptibility $x_{q}$ for APW- and free electrons are given. The numerical accuracy is tested for the free electron model using both a root-sampling method and a linearized integral method. The first method requires 450,000 and the latter 9,000 points in the entire Brillouin zone for a good agreement with the Lindhard function. $x_{q}$ and the wave vector dependent exchange integral $\mathrm{J}_{\mathrm{q}}$ is calculated for $\mathrm{Gd}, \mathrm{Tb}$, Dy, and $\mathrm{Er}$ in the ordered phase. $\mathrm{J}_{\mathrm{q}}$ is calculated for Gd with the inciusion of the matrix element, calculated on the basis of APW functions. 


\section{Exchange Interaction in the Heavy Rare Earth}

Metals Calculated from Energy Bands

by

Per-Anker Lindgard

Danish Atomic Energy Commission

4000 Roskilde

Denmark 


\section{INTEOUVOTION}

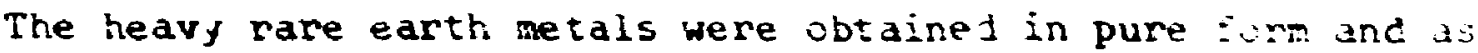
single crystals about ten years ago. This made a detailed experimental investigation possible. Neutron scattering in particular has been an important tool. As a result we by now have obtained a very complete knowledge about the magnetic interactions. The experimental facts, which are reviewed in ${ }^{1)}$, revealed that the magnetic properties are determined by an intricate interplay of forces of similar magnitude. The dominant is the indirect Ruderman-Kittel-Kasuya-Yosida (RKKY) exchange interaction, which we shall attempt to calculate from first principles, here. Of importance is also the crystal field anisotropy and magnetoelastic effects. The anisotropy of this origin is of a single ion type. Recent neutron scattering measurements ${ }^{2}$ ) have shown that also two-ion-anisotropy may be of importance. There are numerous possibilities for anisotropy of the interaction between the moments at different sites. As we shall see the RKKY interaction, which: is mediated by the conduction electrons, is anisotropic in the magnetically orjered phase. The two-ion-interaction, which is mediated by phonons, is strongly anisotropic. The magnitude of the interaction between the spin system and the lattice is determined by the coupling between the spin- and orbital-motion of the electrons. If the spin-orbit coupling and the orbital momentum is large we must therefore expect large arisotropies both of single-ion and two-ion nature. Also the RKKY interaction becomes anisotropic as discussed by Kaplan and Lyons ${ }^{3)}$.

In order to avoid the complications of anisotropy we shali start by considering the RKKY interaction in a pure spin system with no orbital effects. This is examplified by gadolinium, which has $a^{8} \mathrm{~S}$ ground state. The electronic configuration of $\mathrm{a}$ Gd atom is a xenon core with seven $4 \mathrm{f}$ electrons and three ( $5 \mathrm{~d}^{1} \mathrm{fi} \mathrm{s}^{2}$ ) outiver electrons.

The basic interaction is between the localized 4 f electrons belonging to the inner shells of gadolinium and the conduction electrons. Rudermann and Kittel assumed for simplicity that the condition electrons were completely free ( $i . e$. plane wave states). We are now able to go a step further and treat the confuction electrons in a more realistic fashion. A standand techrique 
is the augmented plane wave (APW) method 4,5 ).

\section{THE AUGHENTED PLANE HAVE METHOD (APU)}

In the APU-wethoi the electrons are supposed to wove in a simplified potential which is atomic like inside a sphere (the muffin tin (in two dimensions)) around each ion and constant ( $=0$ ) between the spheres. The Schroding $r$ equation is then solved numerically for this potential by the variational nethod.

The trial wave function is obtained by expanding the wave functions inside the spheres in atonic like functions and between the spheres in plane waves. The vave functions are arched at the surface of the spheres and the coefficients in the expansion is determined by minimizing the energy.

The wave function for the electrons, the crystal uave function, is therefore

$$
t_{\underline{k}, E}(\underline{r})=\sum_{i}^{i} A_{i}(\underline{k}) \underline{k}_{i}(\underline{r}),
$$

Where the coefficients $A_{i}(\underline{k})$ are to be deternined variationally. The sum is over a set of reciprocal lattice vectors $I_{i}$ where we wite $\underline{k}_{i}=\underline{k}+\underline{I}_{i}$.

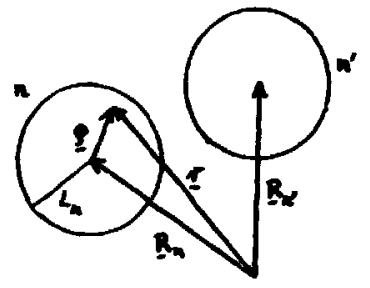

The augwented plane wave is outside the spheres

$$
\phi_{k_{i}}(\underline{r})=\frac{1}{H} e^{i \underline{k_{i}} \cdot \underline{\underline{r}}}
$$

where $\Omega$ is the volume of the unit cell.
Inside the n'th sphere is

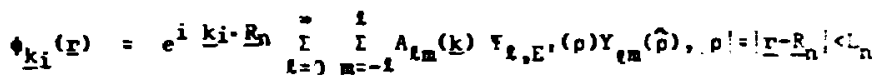

where $L_{n}$ is the radius of the sphere and $R_{n}$ the vector to the center. The number of 2 values to be included in the sula are not specified at this point. However, if we want to represent $s, P$, d or $f$ character of the crystal vave function we must include $l=0,1,2,3$. The wave function $i r$. the two regions (2) and (3) can be made to match at the sphere surface by choosing the expansion coefficients $A_{L_{n}}(\underline{k})$ in (3). By expanding (2) in spherical harmics and Bessel functions around the center of the sphere

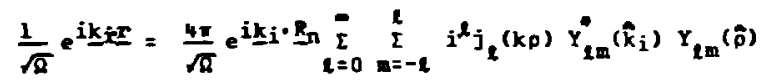

and equating this with ( 3 ) at $p=\mathrm{L}_{\mathrm{h}}$ we find

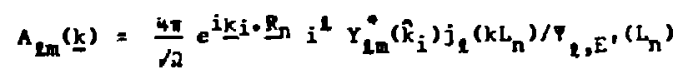

The APW function ${ }_{k_{j}}(\underline{\underline{r}})$ with this $A_{t \rightarrow}(\underline{k})$ is called a basis function. It is continuous, but has a discontinous slope at the sphere radius. The expansion coefficients $A_{i}(\underline{k})$ in $(1)$ are found by minimizing the energy

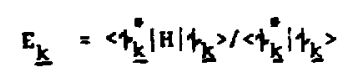

This gives a secular equation for the determation of the $A_{i}(\underline{k})$. we shall not go further into this.

The $r_{1, E},(p)$ functions are the radial solution to the Schrodinger equation inside the sphere

$$
\left(\frac{I}{r^{2}} \frac{d}{d r}\left(r^{2} \frac{d}{d r}\right)+\frac{l(h+1)}{r^{2}}+V(r)-E^{\prime}\right) r_{\ell, E^{\prime}}(r)=0
$$

$T_{2, E},(p)$ must be regular at the center $(p=0)$, but there is no 
boundary condition at $p=m$ and hence there exist solutions for aii $E^{\prime}$. This is a complication and $E^{\prime}$ must be chosen selfconsistently according to (6). Several methods have been devised to wake this practically. Harmon ${ }^{5}$, used a linearized AUP wethod ${ }^{6)}$ to obtain the wavefunctions for $6 d$, which we are going to use later. Also the crystal potential inside the sphere, $V(r)$, must be chosen selfconsistently. This is done by suming the contribution to the coulonb potential from a large numer of surrounding ions including the conduction electron charge density. The exchange interaction may be included in the slater $0^{1 / 3}$ approxination.

By carrying out this programe we are able to find a set of selfconsistent energy bands $E_{k}$ and the correspondint wave functions $t_{k}$ for the conduction electrons. The variationally deternined vavefunctions are presunably less reliable than the energies. Also they are more sensitive to the approximation ade when constructing the muffin-tin-potential. However, we may expect them to be best near the atoms inside the spheres. Therefore they should be quite adequate in calculating the matrix element between the conduction electrons and the localized $4 \mathrm{f}$ electrons, which is relevant for the calculation for the RKXY interaction. The $4 \mathrm{f}$ electrons are well approximated by the atomic wavefunctions of Herman and Skillman ${ }^{7}$.

\section{THE RKKY-INTERACTION HITH} REALISTIC ENERGY BANDS

\subsection{The Interaction between conduction electrons}

\section{and the $4 f$ electrons}

By means of these realistic energy bands and wave functions we can proceed to calculate the ROX interaction. ${ }^{18)}$

In the calculation of the energy bands we did not sonsider explicitly the interaction between two electrons but rather the interaction between one electron and the average potential for all the other electrons. As a perturbation on this model we shall now consider the interaction between a cenduction electron and $\mathrm{a}$ f electron. The direct interaction is the coulomb interaction $v\left(x_{1}-x_{2}\right)=\frac{e^{2}}{\left|\underline{r}_{1}-\underline{r}_{2}\right|}$ betwen a conduction electron at $\underline{r}_{1}$ and a $4 \mathrm{f}$ electron at $\underline{x}_{2}$. In general, however, the potential is screened by the presence of the other electrons, in which case $v\left(\underline{r}_{1}-\underline{r}_{2}\right)$ will be modified to for example the Yukawa potential $e^{2} \exp \left(-x\left|\underline{r}_{1}-\underline{r}_{2}\right|\right) /\left|\underline{r}_{1}-\underline{r}_{2}\right|$, where $k-l_{i s}$ the screening length.

Since we are interested in the agnetic interaction we shall only consider the exchange interaction and further only t..e term which involve the scattering of a conduction electron on a $4 f$ electron.

This is represented in terws of electron creation an 1 annihilation operators, $c_{\underline{k}}^{+}$and $c_{\underline{k}}$ respectively as follows

$$
\begin{gathered}
v\left(\underline{r}_{1}-\underline{r}_{2}\right)=\underset{\substack{\underline{k}_{i} s_{i} \\
i=1,2,3,4}}{\sum}\left\langle\underline{k}_{4} s_{4}, \underline{k}_{3} s_{3}\left|v\left(r_{1}-\underline{r}_{2}\right)\right| \underline{k}_{2} s_{2}, \underline{k}_{1} s_{1}\right\rangle \\
c_{\underline{k}_{4} s_{4}}^{+} c_{\underline{k}_{3} s_{3}}^{+} c_{\underline{k}_{2} s_{2}} c_{\underline{k}_{1} s_{1}}
\end{gathered}
$$

where $|v|>i s$ the matrix elewent, $\underline{k}_{i}$ the crystal momentum and $s_{i}$ the spin index. Since $v\left(\underline{r}_{1}-\underline{r}_{2}\right)$ is independent of spin, the spin ust be conserved in the scattering process. Let us assume that the $4 f$ lectrons are well approximated by localized atomic states ${ }_{4 f}\left(r-R_{n}\right)$ at the site $\underline{R}_{n}$ and the conduction electron wave function is $t_{\underline{k}, E}(r)$ in $(1)$. Then the conduction electrons are scattered from one state of momentum $\underline{k}$ to another of $\underline{k}$ ' whereas the localised electrons are scattered from one localised state to antoher, with or without spin flip. We can represent the change in the localised states by the change in the total local spin $\underline{S}_{n}$ instead of by means of the creation and annihilation operators in (8).

The perturbation of the single electron Hamiltonian which was used in the sand calculation is therefore in this approximation for the $s-f$ cxchange interaction as follows

$$
\begin{aligned}
& H_{g f}\left(\underline{R}_{n}\right)=-\frac{1}{H} \underset{k, k^{\prime}}{\Sigma} j_{B f}\left(k, k^{\prime}\right) e^{i\left(\underline{k}-\underline{k}^{\prime}\right) \underline{R}_{n}} \\
& \left(c_{g}^{+} c_{k^{\prime}}-c_{y^{+}}^{+} c_{k^{\prime} b}\right) s_{n}^{2} \\
& \left.+c_{k}^{+} c_{k^{\prime 2}} 8_{n}^{-}+c_{k+}^{+} c_{k^{\prime}} s_{n}^{+}\right\}
\end{aligned}
$$


where the last line shows the spin flip scattering processes and the middel line the processes without spin flip.

The matrix element is

$$
\begin{aligned}
& j_{s}=\left(\underline{k}, \underline{k}^{\prime}\right)=N \int \underline{d}_{1} \frac{d \underline{r}_{2}}{2}\left(\Phi_{4 f}^{*}\left(\underline{r}_{1}-\underline{R}_{n}\right) \gamma_{\underline{\underline{k}}}^{*}\left(\underline{\underline{r}}_{2}\right) v\left(\underline{r}_{1}-\underline{r}_{2}\right)\right. \\
& \left.\phi_{4 f}\left(\underline{r}_{2}-\underline{R}_{n}\right) \quad \psi_{\underline{k}},\left(\underline{r}_{1}\right)\right\} e^{i(\underline{\underline{k}} \cdot-\underline{k}) \underline{R}_{n}}
\end{aligned}
$$

$j_{s f}\left(\underline{k}, \underline{k}^{\prime}\right)$ is in iependent of the lattice site $\underline{R}_{n}$ since

$$
f_{\underline{k}}(\underline{r})=\underline{u}_{\underline{k}}(\underline{r}) e^{i \underline{k} \underline{r}}=f_{\underline{k}}\left(\underline{r}-\underline{R}_{n}\right)=u_{\underline{k}}(\underline{r}) e^{i \underline{k} \underline{r}} e^{-i \underline{k} R_{n}}
$$

according to Block's theorem. We shall assume that $\phi_{4 f}\left(\underline{r}-\underline{R}_{n}\right)$ vanishes outside the muffin-tin-sphere around $\underline{R}_{n}$ and therefore we only integrate (10) inside the sphere to obtain the geneiralized exchange integral $j_{s f}\left(\underline{k}, \underline{k}^{\prime}\right)$.

\subsection{The effect of orbital moment of the $4 f$ electrons}

Let us generalize the interaction Hamiltonian ( 9 ) sligthly. In the presence of oritial monentum $L$ for the $4 f$ electrons the total angular momentum $\underline{J}=\underline{L}+\underline{S} \quad(J=|L \pm S|$ since $\underline{L}$ and $\underline{S}$ are parallel, with + for the heavy and - for the light rare earth metals). Then we can replace $\underline{S}$ in ( 9 ) by the spin projection along $\mathrm{J}$ nanely $(\mathrm{g}-1) \mathrm{J}$, where $\mathrm{g}$ is the Lande factor. A proper calculation of the orbital effects will give rise to a more complicated form for (9) as discussed by Kaplan and Lyons ${ }^{3)}$. The effect is however small and will be neglected here.

\subsection{The effect of magnetic ordering of the}

localized moments

If the localized moments are ordered througout the crystal they will give rise to a molecular magnetic field $H_{n}$ which will shift the energy of the otherwise degenerate spin-up and spin -down electrons i.e. $E_{k t} \neq E_{k b}$. This molecular field model is equivalent to the rigid-band-shift model. The shift in energies can be calculated exactly by diegonalizing the single electron Hamitonian and the molecular field term

$$
H=\sum_{k, s}^{\sum} E_{k, s} c_{k, s}^{+} c_{k, s}+H_{M}
$$

The molecular field is obtained by taking the thermal average value of localized moments $\underline{s}_{n}$ in (9).

For the sake of generality we shall calculate the RKKY interaction for the conically ordered phase. The cone-structure, which contains as special cases both the ferromagnetic and the spiral structure, is defined by the following parameterization of the ionic moments:

$$
\left\langle\underline{S}_{\mathbb{R}_{n}}\right\rangle=m(T) S\left\{\sin \theta \cos \left(\underline{Q} \cdot \underline{R}_{n}\right), \sin \theta \sin \left(\underline{Q} \cdot \underline{R}_{n}\right), \cos \theta\right\}
$$

where $m(T)$ is the temperature dependent reduced magnetization, $\theta$ is the cone angle and $Q$ the spiral vector.

Using $(9)$ and $(10)$ we find the molecular field $\mathrm{F}_{M}$ to be used in (10), which then can be diagonalized using standard techniques.

\section{We find the new energies}

$$
\varepsilon_{k, Q}^{ \pm}=\varepsilon_{p} \pm \sqrt{\left(\varepsilon_{m}-\Delta\right)^{2}+\gamma^{2}}
$$

where

$$
\begin{aligned}
& \underset{\mathrm{p}}{E_{\mathrm{m}}}=\left(E_{k-Q / 2} \pm E_{k+Q / 2}\right) / 2 \\
& \Delta=S m(T) \cos \theta j_{g f}(k, k) \text { and } \gamma=\operatorname{Sm}(T) \sin \theta j_{s f}(k, k+Q)
\end{aligned}
$$

the new wave functions are

$$
\begin{aligned}
& \psi_{k, \downarrow}=\cos \theta|k-Q / 2, \uparrow\rangle+\sin \phi|k+Q / 2, \downarrow\rangle \\
& \psi_{k,-}=-\sin \theta|k-Q / 2, \uparrow\rangle+\cos \phi|k+Q / 2, \downarrow\rangle
\end{aligned}
$$

where

$$
t g \phi=\frac{x}{\varepsilon_{k, Q}^{+\Delta-E_{k-Q / 2}}}
$$

(12) shows the energies of the conduction electrons in the magnetically ordered phase. 
For the ferromagnetic case $\theta=0$ and $Q=0$ and we find the rigid band model:

$$
r_{k}^{*}=E_{k} \pm \Delta
$$

where $\Delta$ goes to zero when the magnetization vanishes at $\mathbf{T}_{\mathrm{c}}$.

For the spiral case we obtain the results discussed by Elliott and Wedgwood ${ }^{8}$. In this case, as in the general case of cone structure, the magnetic order produces gaps in the electron energy bands related to the spiral vector $Q$. This is of importance when calculating the temperature dependence of the spiral vector $Q(T)$, and in general the temperature dependence of the exchange interaction.

The magnetic order, the effect of which we have just included, is of course a consequence of the interaction between the loca? moments. In other words the interaction must be calculated selfconsistently.

\subsection{The generalized RKKY interaction}

\section{in the ordered phase}

We now proceed to calculate the RKKY interaction by taking into account the terms left in (9). $\mathrm{H}_{\mathbf{s f}}-\mathrm{H}_{M} 13$ not digonal between the states (12), but the effect thereof can be found by second order perturbation theory.

The shift in energy is then using (9) and (12):

$$
\delta E^{(2)}=\underset{n, n+i}{E} \sum_{i}<0\left|H_{s f}\left(R_{n}\right)-H_{M}\right| i>\left\langle i\left|H_{s f}\left(R_{n},\right)-H_{M}\right| O>/\left(\varepsilon_{0}-\varepsilon_{i}\right)\right.
$$

where $|0\rangle,|i\rangle$ are the initial and intermediate states respectively and $\varepsilon_{0}, \varepsilon_{i}$ the corresponding energies, from (12). We must remenber that the electrons can be scattered only from an occupied state to an empty state according to the Pauli principle. This can be accounted for by the fermi factors $f_{k}=\left[e^{\left(E_{k}-\varepsilon_{F}\right) / k T_{+1}}\right]^{-1}$. We shall assume that $f_{k}$ is a step function, being 1 for energies smaller than the fermi energy and 0 for larger snergies. 9 ) We then find from (17) for the cone-structure the following effective interaction between the localized momants.

$$
H_{q}=-J_{q}^{n} s_{q}^{z} s_{q}^{z}-1 J_{q}^{\frac{1}{q}}\left(s_{q}^{+} s_{-q}+s_{q}^{-} s_{-q}^{+}\right)
$$

where the wave vector dependent exchange interaction is

$$
\begin{aligned}
& J_{q}^{\prime \prime} s_{q}^{z} s_{-q}^{z}= \\
& \sum_{n}, s_{n}^{z} s_{n}^{z}, e^{i g\left(R_{n}-R_{n}^{\prime}\right)} \frac{1}{2 N} \sum_{k} f_{k}\left(1-f_{k+q}\right)\left|j_{s f}(k, k+q)\right|^{2} \\
& {\left[\left(1+\cos ^{2} 2 \phi\right)\left(\frac{1}{\varepsilon_{k}^{+}-\varepsilon_{k+q}^{+}}+\frac{1}{\varepsilon_{k}^{-}-\varepsilon_{k+q}^{-}}\right)+\sin ^{2} 2 \phi\left(\frac{1}{\varepsilon_{k}^{+}-\varepsilon_{k+q}^{-}}+\frac{1}{\varepsilon_{k}^{-}-\varepsilon_{k+q}^{+}}\right]\right.}
\end{aligned}
$$

and

$$
\begin{aligned}
& J_{q}^{+}\left(s_{q}^{+} s_{-q}^{-}+s_{q}^{-} s_{-q}^{+}\right)= \\
& \sum_{n n^{\prime}} e^{i q\left(R_{n}-R_{n}{ }^{\prime}\right)}\left\{s_{n}^{+} s_{n}^{-}, e^{i Q\left(R_{n}-R_{n}+\right)}+s_{n}^{-} s_{n}^{+}, e^{-i Q\left(R_{n}-R_{n} \cdot\right)}\right) \\
& \frac{1}{2 N} \sum_{k} f_{k}\left(1-f_{k+q}\right)\left|j_{s f}^{*}(k, k+Q+q) j_{s f}(k, k-Q+q)\right| \\
& {\left[\sin ^{2} 2 \phi\left(\frac{1}{\varepsilon_{k}^{+}-\varepsilon_{k+q}^{+}}+\frac{1}{\varepsilon_{k}^{-}-\varepsilon_{k-q}^{-}}\right)+\left(1+\cos ^{2} 2 \phi\right)\left\{\frac{1}{\varepsilon_{k}^{+}-\varepsilon_{k-q}^{-}}+\frac{1}{\varepsilon_{k}^{-}-\varepsilon_{k+q}^{+}}\right)\right]}
\end{aligned}
$$

For a ferromagnetic ordering is $\phi=\theta=Q=0$ from $(13,15)$ and (19) reduces considerably. We find

$$
\begin{aligned}
& J_{q}^{\prime \prime}=\frac{1}{k} \sum_{k} f_{k}\left(1-f_{k+q}\right)\left|j_{B f}(k, k+q)\right|^{2} \\
& \left\{\frac{1}{\varepsilon_{k}-\varepsilon_{k+q}^{\prime}}+\frac{1}{\varepsilon_{k}-\varepsilon_{k+q}}\right\}
\end{aligned}
$$

and

$$
\begin{aligned}
& \frac{1}{J_{q}}=\frac{1}{N} \sum_{k} f_{k}\left(1-f_{k+q}\right)\left|j_{B f}(k, k+q)\right|^{2} \\
& \left\{\frac{l}{\varepsilon_{k}^{-} \varepsilon_{k+q}}+\frac{1}{\varepsilon_{k}-\varepsilon_{k+q}}\right\}
\end{aligned}
$$

where $\boldsymbol{k}_{k}$ are given by (16). 
This interaction is anisotropic contrary to the paramagnetic RKKY -interaction. For the ferromagnetic phase we obtain a $J_{q}$ and a $J \frac{1}{q}$ for the spin components parallel or perpendicular to the average moment direction. $J_{q}^{\prime \prime}$ involves only scattering of electrons with no spin-flip and $\mathrm{J}_{\frac{1}{q}}{ }^{\mathrm{q}}$ only with spin-flip. J $\frac{1}{\mathrm{q}}$ can be measured directly by $s p i n$ wave measurements, whereas $J_{q}^{\prime \prime}$ cannot be measured as a function of the wave vector. However, the magnetic contribution to the free energy is $-J_{q}^{\prime \prime} s_{q}^{z} s_{q}^{z}$ for $q=0$. If $J_{q}^{n}$ has a maximum for $q \neq 0$ it shows that, if for no other reasons, a nonferromagnetic state would have lower free energy. However it is necessary to calculate selfconsistently the energy difference between the various phases.

\subsection{The magnitude of the $s-f$ interaction}

Experimental information about the magnitude of the $s-f$ interaction $j_{s f}\left(k, k^{\prime}\right)$ can be obtained directly by considering the polarization of the conduction electrons. This can be found either by measuring the total moment $\mathrm{pr}$. atom or by means of NMR technique measuring the magnetic field, which the conduction electrons create at the nucleus.

For ferromagnetic ordering the net polarization is given by the difference between the number of electrons with spin up and spin down. In the rigid band model $(T=0)$ this is to a good approximation

$$
n_{p}-n_{l}=\left(\Delta_{p}-\Delta_{+}\right) \rho\left(E_{F}\right) / 2=\Delta \rho\left(E_{F}\right)=\operatorname{Sm}(T) \frac{1}{N}{ }_{k}^{\Sigma j} g f(k, k) \rho\left(E_{F}\right),
$$

where $\Delta_{f}$ is the energy shift of the spin up and spin down electrons realitive to the paramagnetic fermi-egergy $E_{F}$ and $\rho\left(E_{F}\right)$ is the density of states at the fermi energy. We obtained (21) by averaging over all momenta in (10).

Since each unpaired electron contributes to the magnetic moment by $\frac{1}{2} g_{B} \mu_{B}=1 \mu_{B}$ we find for the average $8-f$ interaction $(m(0)=1)$

$$
j_{B f}(0)=\frac{\Delta}{S}=\frac{2 d H}{8_{g} \mu_{B} S_{p}\left(E_{F}\right)}
$$

where $8 M$ is the conduction electron polarization in $\mu_{B}$ and $g_{B}=2$.
From magnetization data ${ }^{1)}$ and a theory for the temperature dependence of the magnetization ${ }^{10}$ we find the results given in table 1 .

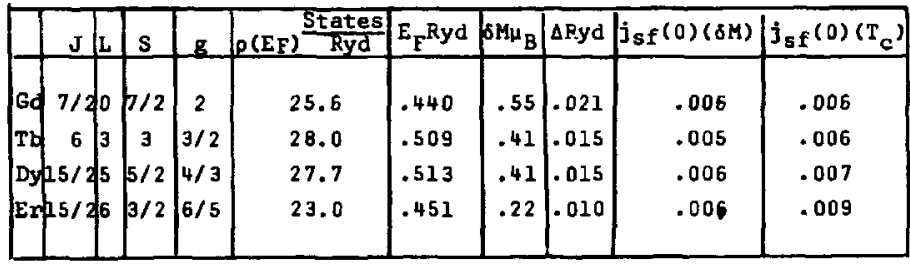

Table 1: Data for the heavy rare earth metals, $\rho\left(E_{F}\right)$ is the calculated density of states, $\Delta$ is half the fermomagnetic splitting and $j_{s f}(0)$, the deduced $s-f$ interaction in Ryd. We notice it is almost independent of the elements. The values estimated from the ferromagnetic transition temperature is given in the last column.

The $s-f$ interaction can be estimated from the ferromagnetic transition temperature as follows

$$
\mathbf{k} \mathbf{r}_{\mathrm{c}}=0.792 \frac{1}{\mathrm{~J}} \mathrm{~J}(\mathrm{~J}+1)
$$

where $J_{0}^{*}=\left[(g-1) j_{g f}(0)\right]^{2} \rho\left(E_{F}\right)$.

0.792 is a factor which corrects the molecular fleld value for $T_{c}$.

Having derived the expressions (19) and (20) for the indirect exchange interaction and estimated the interaction strength we shall consider the actual calculation. The summation over $t\}_{i}-$ wave vectorg $\underline{k}$ in (19) and $(20)$ mugt be done numerically. 


\section{NUMERICAL TETHODS}

On the basis of the APW energy bands calculated by Louks ${ }^{11}$ ) we can evaluate the sums in (19) and $(20)$. The matrix element $j_{g}(k, k+q)$ must be evaluated using the wave functions. The major contribution to the sum comes when the denoninator is small. In other words when the electrons are scattered from just below to just above the fermi surface. This makes the numerical calculation difficult. A possible way is to sum over a very large number of $k$ points and exclude the contribution then the denominator is smaller than a chosen number 6 . This is called the root-sampling method. This is a brute-force principel value calculation (correct in mathematical sense, if we let go to zero). However, it is very difficult to test the convergence of this proceedure numerically. In fact the noise in the computer sets a limit for how small of can be chosen and how fine a mesh of $\underline{k}$ point we can use - apart fron the practical problem of the increasing computer time. However, the method is simple and was used by Liu et al ${ }^{12}$ ) and also in several of the results to be discussed. 13) The convergence seems to be good and the conputing time reasonable with a mesh with 450 060 points in the total Brillouin zone. These calculations were simplified by the assumption that the matrix element $j_{s f}(k, k+q)$ was independent of $k$ and only dependent on the difference $q$, i.e. $j_{s f}(k, k+q) \cdots j_{g f}(q)$.

In order to test the convergence and also to make it feasible to include a $\underline{k}$ dependence of $j_{s f}(k, k+q)$ a different numerical method was used. In this method the Brillouin zone is divided into a relatively small number of micro cells. Inside each cell are the constant energy surfaces $\varepsilon_{k}$ approximated by planes. This makes it possible to integrate analytically inside each micro cell. The integrals are only divergent if the energy surfaces $\varepsilon_{\underline{k}}$ and $\varepsilon_{\underline{k}+q}$ are exactly parallel. This will occur very rarely. "This sôcalled linearized method was developed for density of state calculations by Gilat and Raubenheimer ${ }^{12}$ ) and was later simplified by Jepsen and Andersen, who used it for calculating fermi surface areas. The sum in (19) and (20) are more complicated and has not freviously been calculated using this method. We shall therefore briefly describe it. The Brillouin zone is divided into micro cells of the shape of tetrahedra ${ }^{15}$ ) of a volume $V$ as shown on fig. 1. In each corner are the energies

$$
\varepsilon_{k}^{i}=\varepsilon_{1}, \varepsilon_{2}, \varepsilon_{3}, \varepsilon_{4} \text { and } \varepsilon_{k+q}^{i}=e_{1}, e_{2}, e_{3}, e_{4}
$$

Since the constant energy surfaces are approxinated by planes the constant energy difference $y=\varepsilon-e$ is also a plane. The probien is therefore to integrate

$$
I=\int_{0}^{\max } \frac{1}{\min } A(\omega) \mathrm{d} \omega
$$

over the part $P$ of the tetrahedra for which $C<E_{F}$ and $e>E_{F}$, where the area of the constant energy difference plane inside $P$ is $A(*)$. $P$ may be a complicated polyhedra because of the restrictions coning fron the fermi $f_{k}$ factors in (19) and (20). $f_{k}\left(1-f_{k+q}\right)$ can by syetry considerations be replaced by $\mathbf{l}\left(f_{k}-f_{k+q}\right)$. We do not use the latter fore (although it simplifies the calculation considerably) because the result then is given as the difference between two large nubers which may be inaccurate nuerically. For illibtration whall consider the case where the condition $\left(c<E_{F}\right.$ and $\left.e>E_{F}\right)$ is fulfilled for the whole tetrahedra.

The area $A(\omega)$ is then simply the area of a cut of the tetrahedra perpendicular to the - planes. This area is clearly a quadratic function of $\omega$, being zero for $\omega$ outside the range $\omega_{\text {max }}$ -w $w$. The area can easily be expressed by geometricul considerations in teres of the comer energies $e^{i}$ and $e^{i}$ and $v$, it is not necessary to calculate the normal vector to the plases. Therefore, in the case where we must integrate over the whole tetrahedre (23) is simply

$$
I=\Sigma \int_{\omega_{\min }}^{\max }\left\{a_{n}\left(\varepsilon^{i} e^{i}\right)+b_{n}\left(\varepsilon_{,}^{i} e^{i}\right) \omega+c_{n}\left(\varepsilon^{i}, e^{i}\right) \omega^{2}\right\} / \omega d \omega,
$$

where the su is over each type of cross sections (triangle or square) and $a_{n}, b_{n}, c_{n}$ the paraneters characterizing this, We notice that the integral is logariteicly divergent when "aln ${ }^{2}$ nax 1.e. when the plenes of $c_{k}$ and $c_{k+g}$ are parallel.

We can test the whod on the Iree electron wodel where the enercy bend: are parabolic. The su $(19,20)$ cen then be integrated exactly giving the Lindhard function. The result of the root- 
sampling mothod and the linearized method is show on fig. 2 together with the exact result. We see that the linearized method gives an excellent result for only $9000 \mathrm{k}$-points in the entire Brillouin zone.

\section{RESULTS}

Let us start by considering what effect the magnetic ordering has on the RKKY exchange interaction. That is the same as asking what is the intrinsic temperature dependence. The formulas were developed in ( 15 ) and (20). We shall only be interefted in a qualitative answer, which will show the general magnitude and the direction of the effects. We therefore make the simplifying assumption that for this purpose we can consider the matrix element $j_{s f}(k, k+q)$ to $\mathrm{cnly}$ depend on the difference $q$. Our problem then reduces to calcuiating the electronic static susceptibility.

$$
x_{q}^{\alpha \beta} \sim \frac{1}{N} \sum_{k} F_{k}\left(1-f_{k+q}\right) /\left(E_{k}^{\alpha}-\varepsilon_{k+q}^{\beta}\right)
$$

We determise the matrix element $\left|j_{s f}(q)\right| 2$ from experiments, by comparing $x_{q}^{\frac{1}{q}}$, the calculated sum without it, with the $J \frac{1}{q}$ obtained from spin wave measurements. The matrix element is assumed to be insensitive to the magnetic structure and is used in obtaining the exchange interaction in other magnetic phases. The absolute scale of $\mathrm{J} \frac{1}{\mathrm{q}}$ cannot be determined from the opin waves. The scale is found from the transition temperature $T_{N}$ and coincidently from the conduction electron polarization table 1 . This gives $J$ for $q=0$.

Fig. 3 shows the results for the ferromagnetic phase at $T=0$ for Gd, Tb, Dy, and Er using the APW energy bands and the root -sampling method with 450000 points in the entire brillouin-zone (the linearized method was also used as a test, it gave essentially the identical result and is not shown). It is clear that $\mathrm{Jq}_{\mathrm{q}}^{\mathrm{m}}$ and J $\frac{1}{a}$ differ significantly for all materlals. The dots show the points compared with the experimentel $J_{\frac{1}{a}}$; the calculation was done for 60 equidistant q-values. For terbium the experinental jt shows no naximum for $q \neq 0$, whereas the calculated $J_{q}^{\prime \prime}$ shows that $T b$ has a tendency to form a spiral structure even in the ferromagnetic phase. The enhancement of the maximum for $q \neq 0$ is also evident for Dy and $E r$ in which the spiral region is large. The opposite effect occurs for Gd, where $J_{q}^{n}$ shows that Gd should not form a spiral phase, and nor it does. Furthermore it is clear that the maxima in $J_{q}^{\prime \prime}$ occurs at $q$-values very close to the experimental spiral vectors (indicated with an arrow) and that it is significantly displaced from the peaks in $x(q)$, which is directly related to the presence of flat parallel pieces of Fermi surface. The matrix elenent thus plays an important role in detemining the wave vector dependence of the exchange interaction. The semiemperically found wave vector dependence of the matrix element is very similar for all materials, despite the rather different $x(q)$ functions. This is encouraging for the present analysis. Overhauser ${ }^{16)}$ has argued that the matrix element should follow the 4f-form factor. By extending his model to include the Bloch character of the conduction electrons we would expect a narrow central peak originating from the conduction electrons. This is the form found in fig. 4.

The energy difference between the ferromagnetic and spiral phases is, as judged from the $T=0$ fermomagnetic data fig. 4, for $\mathrm{Gd}, \mathrm{Tb}, \mathrm{Dy}$, and $\mathrm{Er}$ in per cent of the exchange energy: $-148,+58$, +58, +124. This gives for $\mathrm{Tb}$, Dy, and Er a stabilization of the spiral phase by $10 \mathrm{~K} /$ ion times the reduced magnetization squared. The nagnetoelastic stabilization of the ferromagnetic phase is for these naterials at the ferromagnetic-spiral transition typically $1 \mathrm{~K} / \mathrm{ion}$.

The last column in fig. 4 shows a calculation at half the saturation moment of $x_{0}^{n}(q)$ in the ferromagnetic phase and $x_{q}(q)$ in the spiral phase, with spiral vector $Q$. The contribution to the free energy is proportional to $-|j(Q)|^{2} \times f(0) \cdot x \frac{f}{0}(0)$ as a function of the epiral vector $Q$ follows closely that of $x_{0}^{\pi}(q)$ as a function of $\mathrm{q}$, which shows that the most probable spiral vector colncide with that found in the ferromagnetic phase. The precise location is sensitive to the wave vector dependence of the matrix element.

The above simple calculation gave encouraging results and is a natural extension of the calculation of the exchange interaction in the paramapnetic phace. ${ }^{12}$ ) However, the next step is 
to consider the eatrix element wore seriously. We shall do this for the paranagnetic phase with no band splitting. Harmon (5) has by weans of the APG functions calculated $\left|j_{s f}(k, k+q)\right|^{2}$ for the simplest material $G$. A few of the matrix elements are shom on fig. 3. They generally show the q dependence we anticipated, nancly a sharp peak at $q=0$. On the other hand it is clear that they are quite sensitive to the value of $\underline{k}$, and irregularities occur as a function of $q$, which presuably comes fron the hybridization of the $p-$ and d-wave functions.

It is therefore of importance to carry out the complete su (20) including the $k$ dependence of $j_{s}(\underline{k}, k+q)$. Preliminary reaults are show on fig. 5. The calculation is perfored bf the linearized-integral wethod (23) with $7000 \mathrm{k}$ points in the entire Brillouin zone and with $j_{s f}(\underline{k}, \underline{k}+q)$ included rectangularily at $1250 \mathrm{k}$ points. The result is the first direct calculation of the RKKY interaction for Gd with no adjustable parameters. The $q$-dependence of $J_{q}$ is in satisfactory agreenent with that obtained experimentally from spin vave easurements, shown as $\mathrm{J} \frac{1}{\mathrm{~g}}$ in fig. 4 . An important question to be investigated is if the major contribution to $J_{q}$ cones fron the part of the sw for wilh $j_{s f}(\underline{k}, \underline{k}+q)$ is insensitive to $\underline{k}$ or if both the $\underline{k}$ and $\underline{q}$ dependence are equally important, the last case would indicate that the matrix element is as important in determining the magnetic properties of the heavy rare earths as the fermi surface topology.

Work on these questions is in progress. A large number of problems are waiting to be dore in developing and refining the theory, here presented, and confronting it with the experimental facts.

\section{REFERENCES}

1) K.J. Elliott ad., Magnetic Properties of Rare Earth Metals (Plenum Press, London, 1972)

2) J.6. Houran, J. Jensen, F. Touborg (iy74) to be published

3) J.A. Kaplan and D.H. Lyans, Phys. Rev., 129, 2072 (1963)

4) T.i. Louks, Augented Plane Wave Method (H.A. Benjanin, Inc., Hew York, 1967)

5) B.A. Harmon, Conduction Electron Polarization, Spin Densities and The Heutron Magnetic Formfactor of Gadolinium. Thesis (1973)

6) D.D. Koelling, J. Phys. Then. Sol, $\underline{33}, 1335$ (1972)

7) F. Herman and S. Skillan, Atonic Structure Calculations, Prentice-Hall, Inc. Englewood Cliffs, New Jersey (1963).

8) R.J. Elliott and F.A. Hedgwood, Proc. Phys. Soc., 81, 046 (1963)

9) This is a very good approximation at all relevant temperatures.

10) P.A. Lindgind and O. Danielsen (1974) (to be published)

11) S.C. Keeton and T.L. Louks, Phys. Rev. 168, 672 (1968)

12) S.H. Liu, R.P. Gupta, S.K. Sinha, Phys. Rev. B, 4, 1100 (1971)

13) P.A. Lindghrd and S.H. Liu, Proceeding of the Int. Conf. on Magnetise, Moscov (1973).

14) 6. Gilat and L.J. Raubenheimer, Phys. Rev., 144, 390 (1966)

15) 0. Jepsen and 0.K. Andersen, Solid State Commun., 9, 1763 (1971)

16) A.W. Overhauser, J. Appl. Phys., 34, 1019 (1963)

17) W.E. Everson and S.H. Liu, Phys. Rev., 178, 783 (1968)

18) We follow closely the notation of A.J. Freeman ref. 1. 


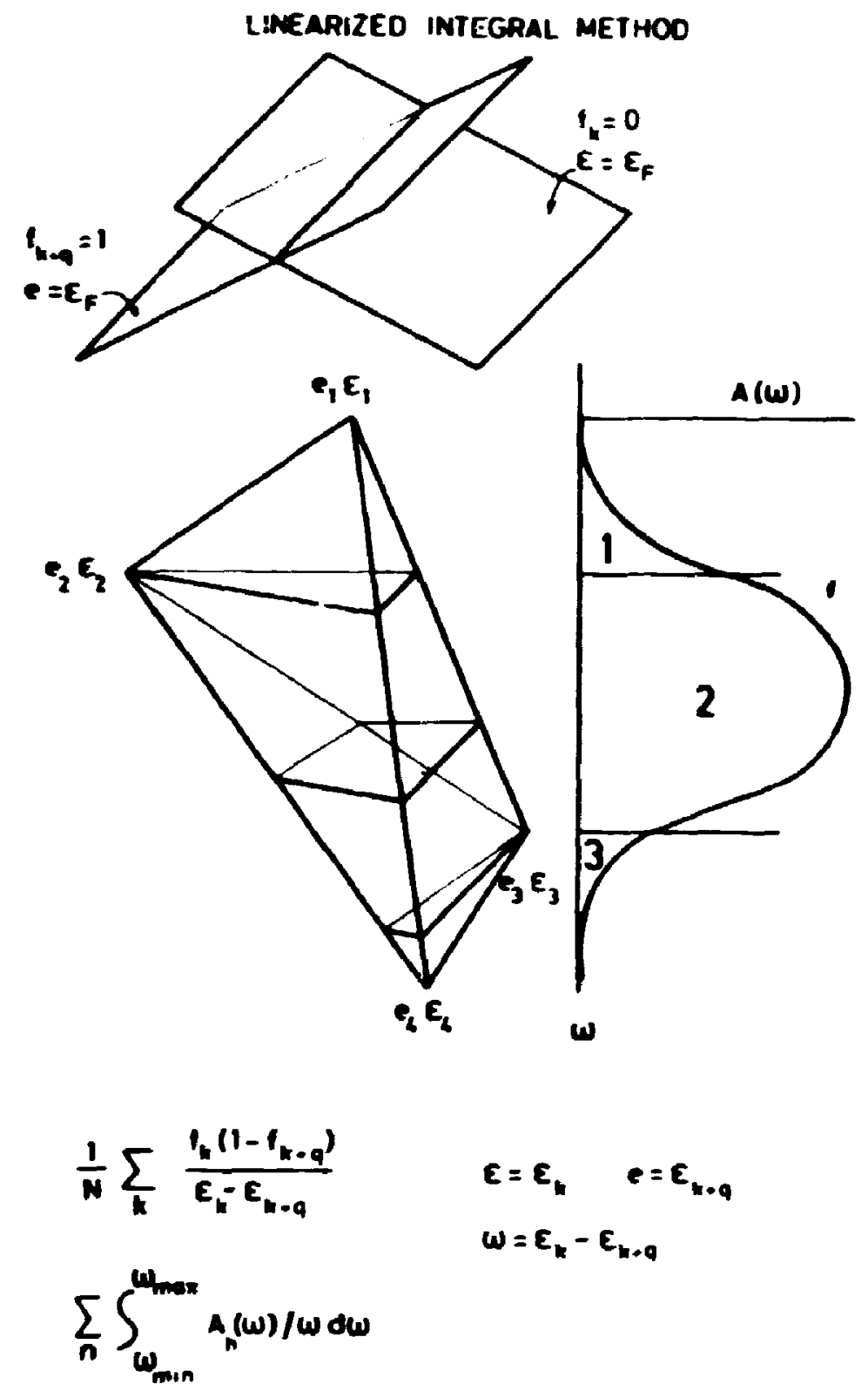

Fig. 1 At the top is shown the constant energy plane, for $\varepsilon_{k}=\varepsilon_{F}$ and $\varepsilon_{k+q}=\varepsilon_{F}$. The Brillouin zone is divided into tetrahedra as show below of constant volume $V$, here oriented so that the direction of increasing energy difference $w$ is vertical. The cut with the constant planes are shown. The area of these cuts are quadratic functions of $\omega$ in the regions 1,2 and 3 . The sum then reduces to the integral show in the lowest line. In general the plane $\varepsilon_{k} \varepsilon_{F}$ and $\varepsilon_{k+q}{ }^{2 \varepsilon_{F}}$ may also cut the tetrahedra. In this case must only be integrated over the part $P$ for which the $f_{k}\left(1-f_{k+q}\right)$ condition is fulfilled. 

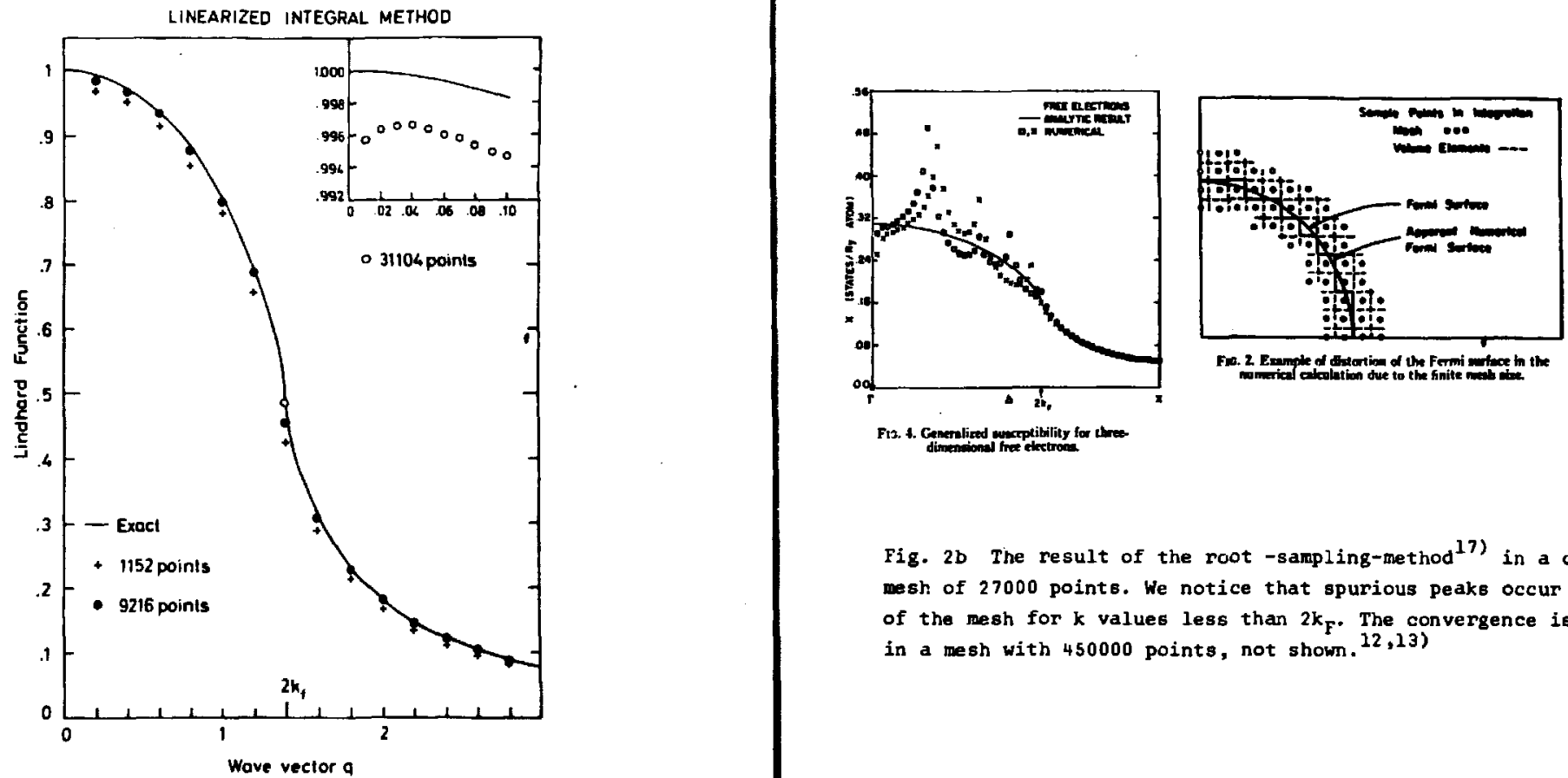

Fig. 2b The result of the root-sampling-method ${ }^{17)}$ in a coarse mess: of 27000 points. We notice that spurious peaks occur because of the mesh for $k$ values less than $2 k_{F}$. The convergence is good in a wesh with 450000 points, not shown. 12,13 )

Fig. 2a The generalized susceptibility for free electrons. The points are the numerical results for the linearized-integral -method for meshes with 1000,9000 and 30000 points in the entire Brillouin zone (hcp) with $k_{F}=0.7$ of the zoneboundary wavevector $(\Gamma-K)$. We notice a very good agreement with the theoretical Lindhard function already with the mesh with 9000 points. The insert shows that the most difficult region for $q+0$ is reprocuced we11. The oystematic deviation is due to the fact that the integration is performed in the inscribed polyhedra in the formi sphere. It has both convex and concave parto and the volum is better approximated by the polyhedra in a realistic syetom. 


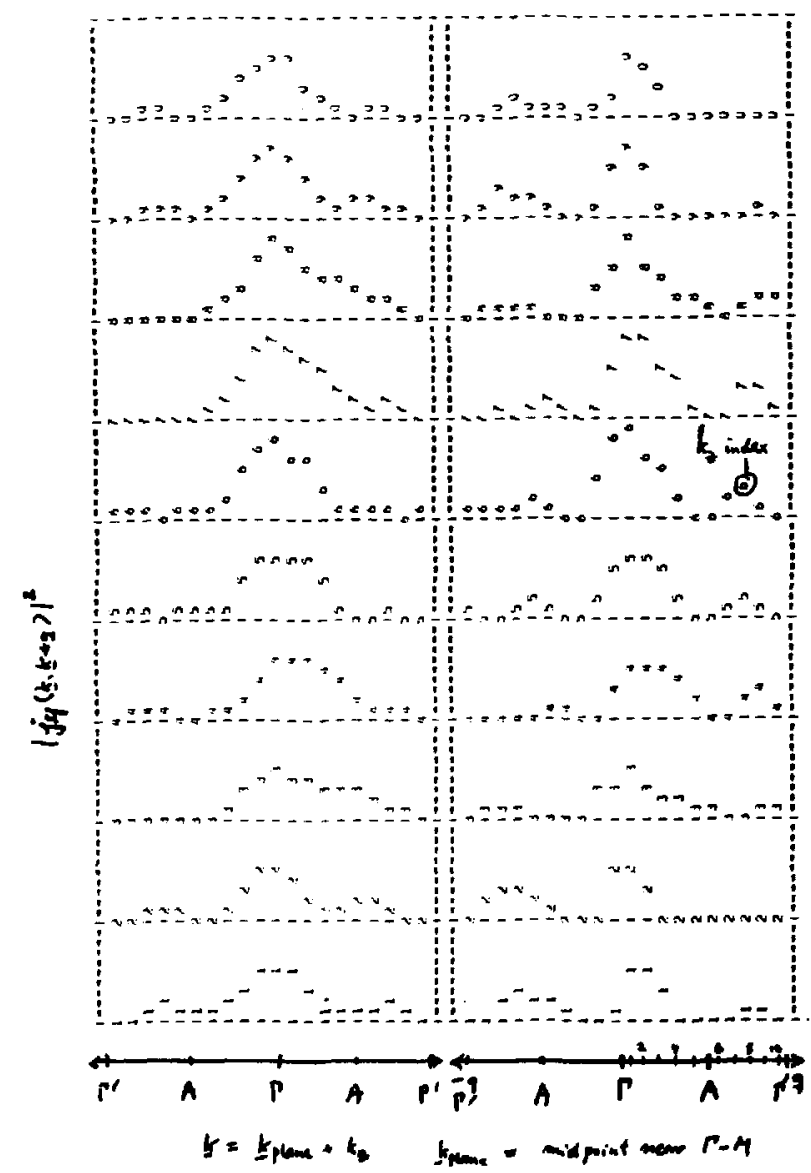

Fig. 3 Example of $\left|j_{g f}(k, k+q)\right|^{2}$ for Gd calculated by the APW method by Harmon. ${ }^{5}$ )
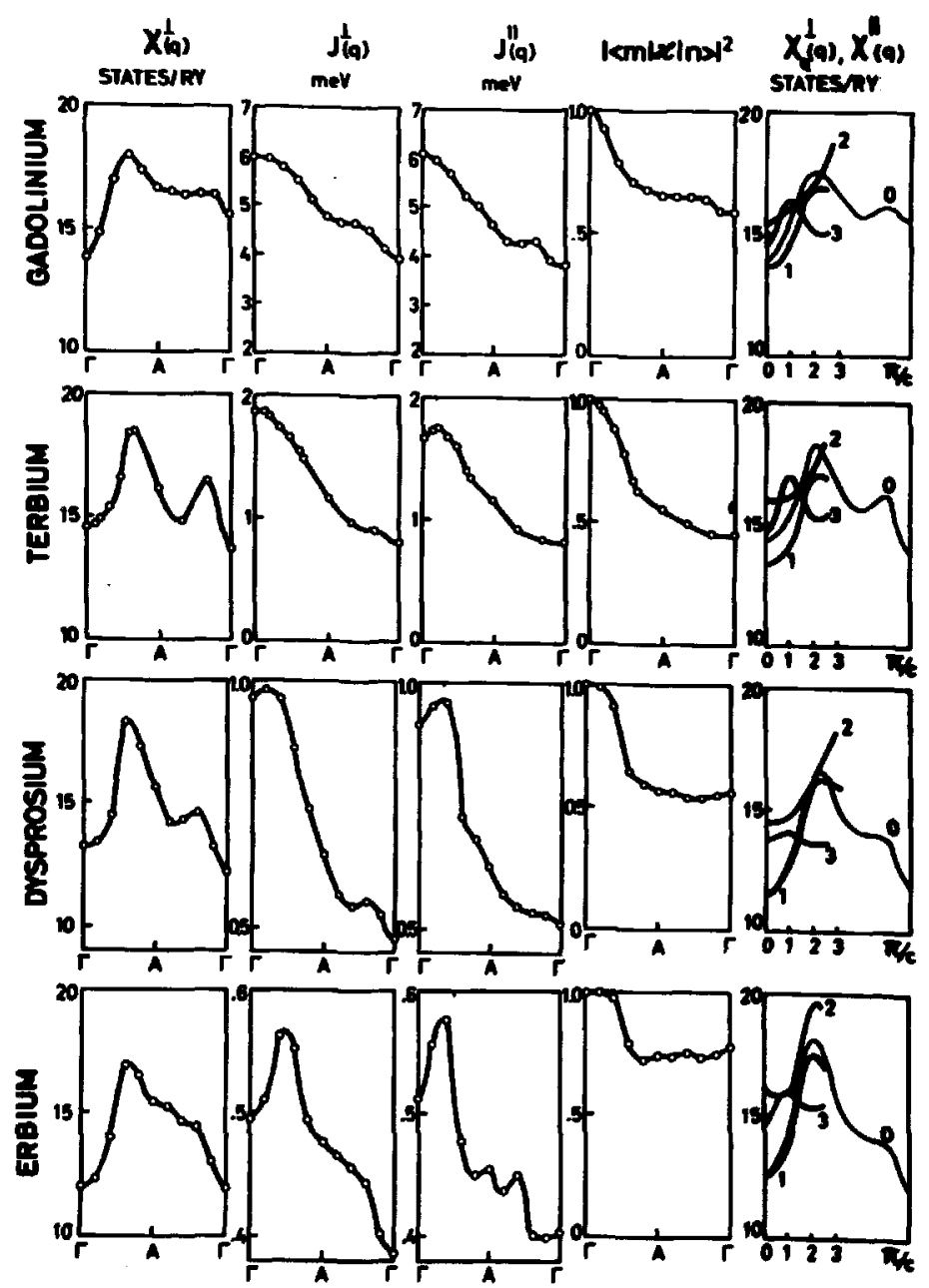

Fis. 4. The perpendicular susceptibility $\left.x_{(q)}^{(}\right)$, the perpendicular experimental exchange interaction $J \frac{1}{q}$, the calculated parallel exchange interaction $J_{q}^{\prime \prime}$ and the deduced matrix element $|j(q)|^{2} /|j(0)|^{2}=$ $|<m| x|n>|^{2}$ for the ferromagnetic phase (splitting: 0.008 Ryd). The last column shows $x f(q)$ in the opiral phase for $Q_{0}=0, Q_{1}=\frac{1}{6} \frac{1}{c}$, $Q_{2}=\frac{2}{6} \frac{1}{c}$ and $Q_{3}=\frac{3}{6} \frac{\pi}{6}$ (aplitting 0.004 Ryd); the corresponding ferromagnetic $x^{\prime \prime}(q)$ is aloo ohown (thin line). 

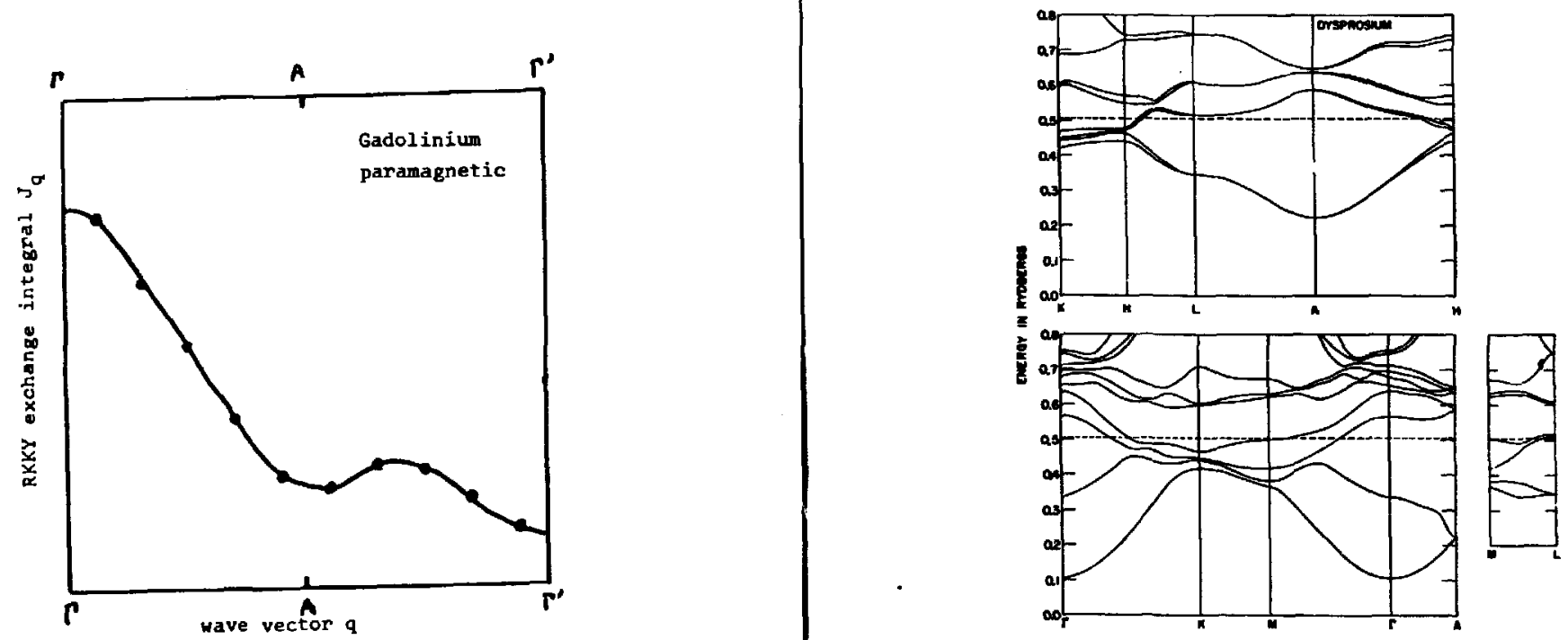

Fig. 5. Preliminary result for the calculated RKKY interaction, using Harmon's APW matrix element ${ }^{5)}$. Only scattering relevant for an extended zone has been included as a first approximation.

Fig. 6. APW energy bands for $D y^{11)}$. Only the bands crossing the fermi surface have been included in the sum. 